\title{
p53-Fibrinolytic system and acute lung injury
}

\author{
Yashodhar Prabhakar BHANDARY* \\ Yenepoya Research Centre, Yenepoya University, Deralakatte, Mangaluru - 575018, India; \\ e-mail: yash28bhandary@gmail.com
}

\begin{abstract}
A different form of lung disease including acute lung injury (ALI) and its most severe form, acute respiratory distress syndrome, bronchiolitis, interstitial lung diseases and drug-induced lung diseases are often associated with alveolar epithelial cell apoptosis. Epithelial cells that are the prime important cell in the alveolar architecture produce fibrinolytic components, such as urokinase-type plasminogen activator (uPA), its receptor (uPAR), plasminogen activator inhibitor-1 (PAI-1), and tumor suppressor protein p53. The increased expression of p53, which is responsible for apoptosis of alveolar epithelial cells, and the other components of the fibrinolytic system, and a decreased alveolar fibrinolysis, are strongly involved in the pathogenesis of ALI. The fibrinolytic system, such as uPA, uPAR and PAI-1 interaction with p53, brings about the regulation of the signaling response, as well as the fibrinolytic properties, which will be useful in maintaining the unity of the cell, and also providing the signals to the cells on whether they undergo apoptosis or survival after ALI.
\end{abstract}

Key words: acute lung injury; epithelial cell apoptosis; p53; urokinase plasminogen activator.

Abbreviations: ALI, acute lung injury; ATII, alveolar type II; Bcl-X $\mathrm{L}_{\mathrm{L}}$, B-cell lymphoma-extra large; BLM, bleomycin; CS, cigarette smoke; CSP, caveolin scaffolding domain; PAI-1, plasminogen activator inhibitor-1; uPA, urokinase-type plasminogen activator; uPAR, urokinase plasminogen activator receptor; UTR, untranslated region.

\section{Introduction}

Lung is the essential respiratory organ in many airbreathing animals. The alveolar and the airway compartment of the lung are often exposed to various agents (Bhandary et al. 2012). During normal process, the fibrin deposition in the lung is prevented mainly through efficient operation of the fibrinolytic system (Bhandary et al. 2012). Urokinase-type plasminogen activator (uPA) forms the important component of fibrinolysis, which is produced by the epithelial cells of the lung and is a $55 \mathrm{kDa}$ serine protease stimulated by proinflammatory cytokines (Shetty et al. 2008a). The main function of this protease is to cleave plasminogen to plasmin and degrade the extracellular matrix components (Drapkin et al. 2000). The receptor form of $\mathrm{uPA}$, urokinase plasminogen activator receptor ( $\mathrm{UPAR})$ expressed by lung epithelial cells is also involved in many biological processes (Shetty et al. 2008a). The inhibitors of urokinase, namely plasminogen activator inhibitor-1 (PAI-1) and PAI-2, the two major endogenous plasminogen activator inhibitors produced by the alveolar epithelial cells play an important role in fibrinolysis (Shetty et al. 2008a).

Acute lung injury (ALI) is often bound with considerable apoptosis of alveolar epithelial cells. Increased expression of p53 resulting from DNA damage and defective fibrinolysis due to increasing in the PAI-1 is one of the prominent features of ALI. These events are seen frequently during cigarette smoke (CS) exposure (Shetty et al. 2012), bleomycin (BLM) (Bhandary et al. 2012, 2013), and silica-induced lung injury (Bhandary et al. 2015a). Fibrinolytic defect due to decreased uPA activity is always seen in ALI or its most severe form acute respiratory disease syndrome (Chapman 1995; Idell 1994, 1995, 2002; Horwitz et al. 2008) or interstitial lung diseases (Bertozzi et al. 1990; Idell 1994, 1995, 2002; Olman et al. 1995; Barzonne et al. 1996; Eitzman et al. 1996; Sisson et al. 1999; Tiddens et al. 2000; Jeffery et al. 2001; Chapman 2004; Shetty et al. 2007; Horowitz et al. 2008; Shetty et al. 2012; Bhandary et al. 2015a). The mitogenic nature of uPA enhances the plasminogen activation in alveolar epithelial cells. This response occurs through post-transcriptional stabilization of uPA, uPAR, and PAI-1, and extensive cross-talk with p53. Post-transcriptional stabilization is mainly through binding of p53 at $35^{\text {th }}, 37^{\text {th }}$ and $70^{\text {th }}$ nucleotide of the 3' untranslated region (UTR) sequence of uPA, uPAR and PAI-1 mRNA, respectively, and regulates these proteins (Shetty et al., 2008, Shetty et al., 2007, Shetty et al., 2008b). The combined interaction of uPA, uPAR, and PAI-1 controls the viability of lung epithelial and carcinoma cells (Shetty et al. 2007, 2008a,b; Shetty et al. 2008). While induction of proliferation depends on suppression of p53 (Shetty \& Idell 2000; Alfono et al. 2005; Shetty et al. 2005, 2006, 2007, 2008b; Shetty et al. 2008), lung epithelial cell viability is mediated mainly through uPA (Shetty \& Idell 2000; Shetty

\footnotetext{
* Corresponding author
} 


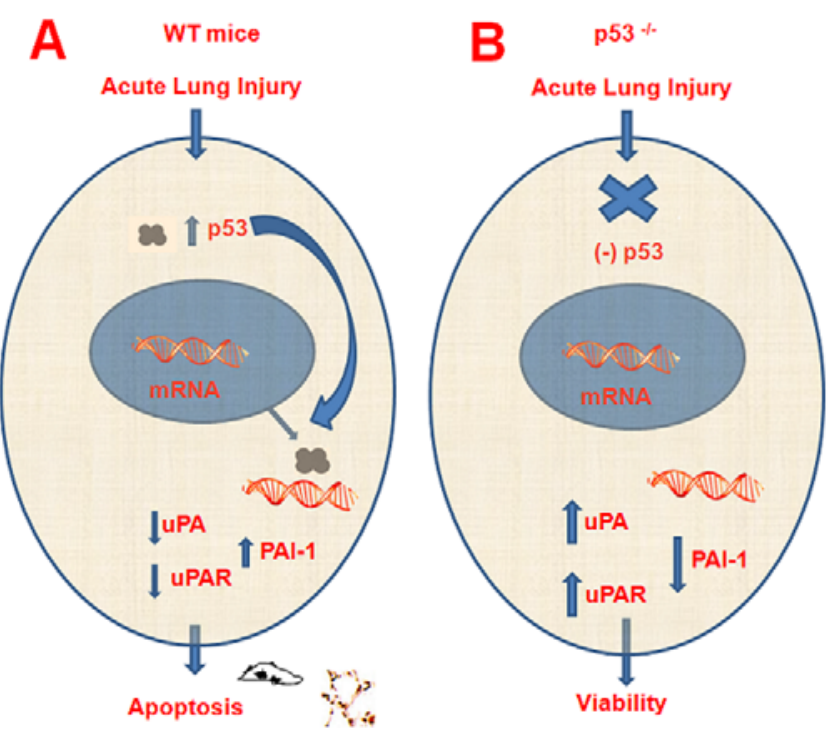

Fig. 1. Model illustration of alveolar epithelial cell apoptosis through cross-talk between p53 and components of fibrinolytic system. (A) p53 is induced during ALI, binds transcripts for uPA, uPAR, and PAI-1 and inhibits uPA and uPAR mRNA through destabilization of the transcripts. PAI-1 is induced through p53mediated stabilization of PAI- 1 mRNA, the end result is reciprocal suppression of uPA and UPAR and increased PAI-1 expression by ATII cells. (B) In p53-deficient mice, in the absence of p53, there will be stabilization of uPA and uPAR mRNA and destabilization of PAI-1 mRNA, which results in increased expression of $\mathrm{UPA}$ and $\mathrm{uPAR}$ and suppression of PAI-1 thereby resulting in the cell viability.

et al. 2005, 2007). Literature shows that healthy alveolar type II (ATII) cells, transplanted to injured lung results in the diminution of apoptotic ATII cells, indicating that ATII cell apoptosis plays a major role during ALI (Serrano-Mollar et al. 2007), i.e. in a process, where maintaining the ATII cell viability through inducing cell proliferation or by inhibiting cell apoptosis could control lung injury. ATII cells that express uPA, PAI-1 and p53 proteins, are responsible for all the major activities applicable to ALI and repair including injunction of lung epithelial cell viability.

\section{p53 and ALI}

Studies showed that p53 protein present in the alveolar epithelial cells is involved in regulatory mechanisms that are accountable for ALI. The maximum induction of p53 expression is seen within 5-7 days in mice upon exposure to BLM (Drapkin et al. 2000; Shetty et al. 2008a) or CS (Bhandary et al. 2012; Shetty et al. 2012) or particulate matter (Shetty et al. 2012; Bhandary et al. 2015a,b). p53 binds to uPA, uPAR and PAI-1 mRNA and brings about destabilization of $\mathrm{UPA}$ and $\mathrm{UPAR}$ mRNA and stabilization of PAI-1 mRNA, thereby decreasing $\mathrm{UPA}$ and $\mathrm{UPAR}$ at the protein and mRNA levels, and increasing the expression of PAI-1 (Shetty et al. 2007, 2008a,b; Shetty et al. 2008). This results in the extensive apoptosis of alveolar epithelial cells (Fig. 1).

ATII cell apoptosis has been studied in var- ious animal models. Elevated ATII cell apoptosis in transgenic mice expressing transcriptionally inert DNp53 (Bhandary et al. 2013) and negligible effect of pifithrin- $\alpha$ on $\mathrm{uPA}$ and PAI-1 mRNA expression levels indicate that p53 alters the expression of $\mathrm{uPA}$ and PAI-1 at the posttranscriptional level, independent of p53 transcriptional activity (Bhandary et al. 2013). This is further supported by a study involving a peptide derived from caveolin-1. Caveolin-1 is expressed by many cells and contains a modular protein domain called caveolin scaffolding domain (CSP). This protein inhibits the tyrosine/threonine kinases (Lisanthi et al. 1995; Wei et al. 1999; Liu et al. 2001; Wanaski et al. 2003). Intervention studies using CSP help in mitigating ATII cell apoptosis in the BLM or CS-exposed mice (Bhandary et al. 2012; Shetty et al. 2012). This is mainly due to inhibition of p53 and PAI-1 expression and induction of uPA and UPAR expression by CSP in the ATII cells (Bhandary et al. 2012, 2013, 2015a; Shetty et al. 2012). These changes prevent the ATII cells becoming apoptotic, indicating that the reversal of p53-mediated changes in fibrinolytic system contribute to the prevention of apoptosis during ALI (Bhandary et al. 2012, 2013, 2015a,b; Shetty et al. 2012). The further nullified response of 3'UTR chimeric sequence to BLM exposure specify that the cell can retain the uPA expression at the normal level followed by the reduction of PAI-1 and complete suppression of caspase-3 activation (Bhandary et al. 2012, 2013, 2015a; Shetty et al. 2012). 3'UTR chimeric sequence suppressed ATII cell apoptosis validates the contribution of cross-talk between p53 and the fibrinolytic system in defining the extent of ATII cell damage.

The increase in p53 is also associated with elevated levels of the pro-apoptotic protein Bax in the passive CS-exposed lung, and inhibition of its expression after CSP treatment show that Bax can contribute to p53-induced ATII cell apoptosis (Shetty et al. 2012). Bax is a proapoptotic member of the Bcl-2 cell death family; Bax transcription is regulated through the Bax gene promoter region, which contains p53 consensus sequences (Miyashita et al. 1994, 1995; Shetty et al. 2012). Though the above fact is possible in most of the study, this effect is abrogated as PAI-1-deficient mice also resisted ATII cell apoptosis, with the response similar to that of p53 deficient mice (Fig. 1). This clearly shows that there are other intermediaries of apoptotic pathways contributing in the process of apoptosis (Shetty et al. 2012). Up-regulation of B-cell lymphoma-extra large $\left(\mathrm{Bcl}_{\mathrm{L}}\right)$ by CSP shows the involvement of Bcl- $\mathrm{X}_{\mathrm{L}}$ in protecting against the ATII cell apoptosis (Bhandary et al. 2012; Shetty et al. 2012) (Fig. 2). Further, the activation of caspase-3 through p53-mediated pathway shows the important role played by p53 in the alveolar epithelial cell apoptosis during ALI.

\section{uPA and ALI}

The proinflammatory cytokines, in particular, tumor necrosis factor- $\alpha$ and transforming growth factor- $\beta$ reg- 

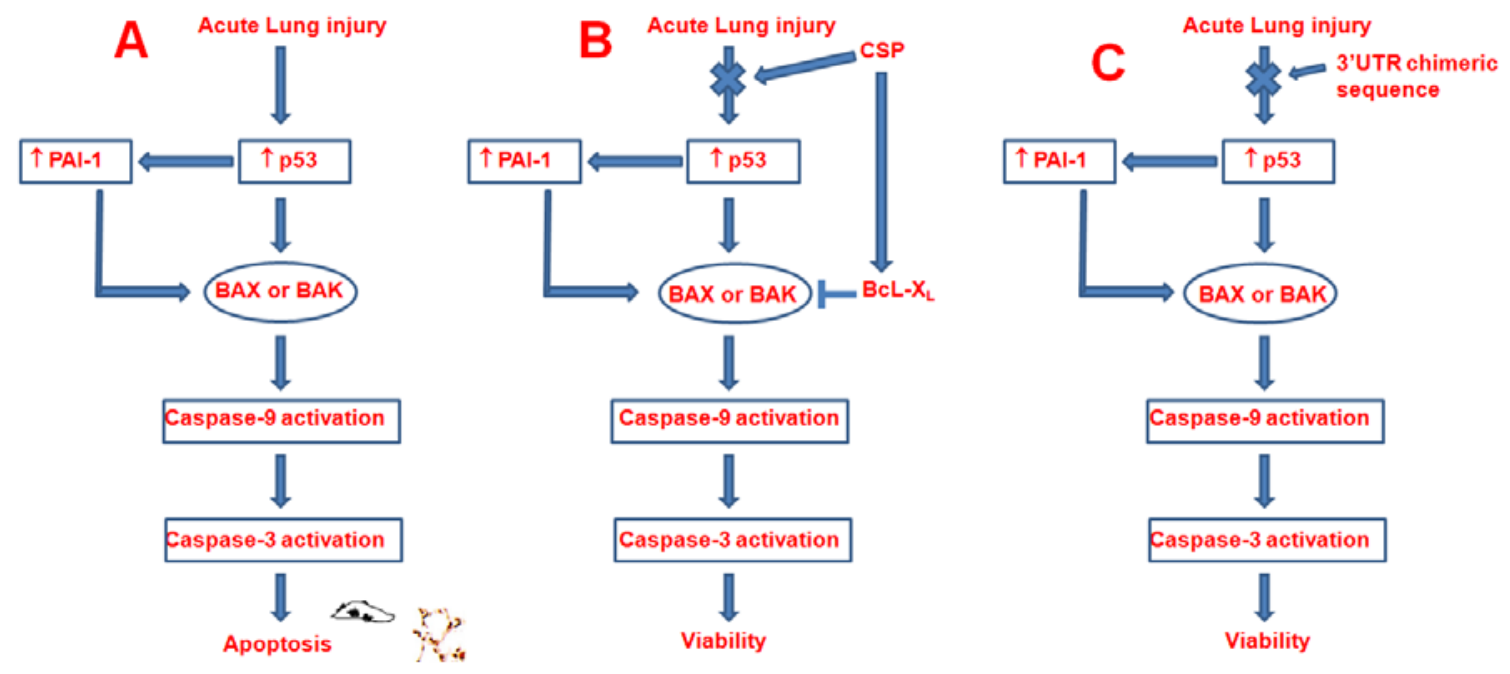

Fig. 2. p53-mediated caspase-3 activation in wild-type mice. (A) In response to DNA damage, p53 activates cell death via BAX or BAK activation, leading to cytochrome $c$ (cyt $c$ ) release, which in turn activates caspase- 9 and initiates the caspase activation cascade. (B) Expression of p53 can be inhibited by intervention through CSP. This intervention also up-regulate Bcl- $\mathrm{X}_{\mathrm{L}}$ and brings about the protection of alveolar epithelial cell apoptosis. (C) p53 binding 3'UTR chimeric sequence controls p53-mediated PAI-1 expression and ATII cell apoptosis by interfering with p53 and PAI-1 mRNA expression.

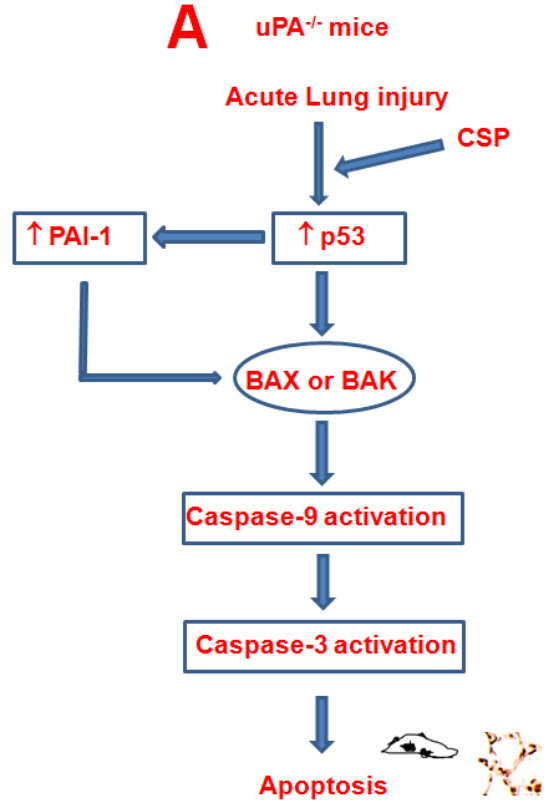

8 uPA $^{-1-}$ mice

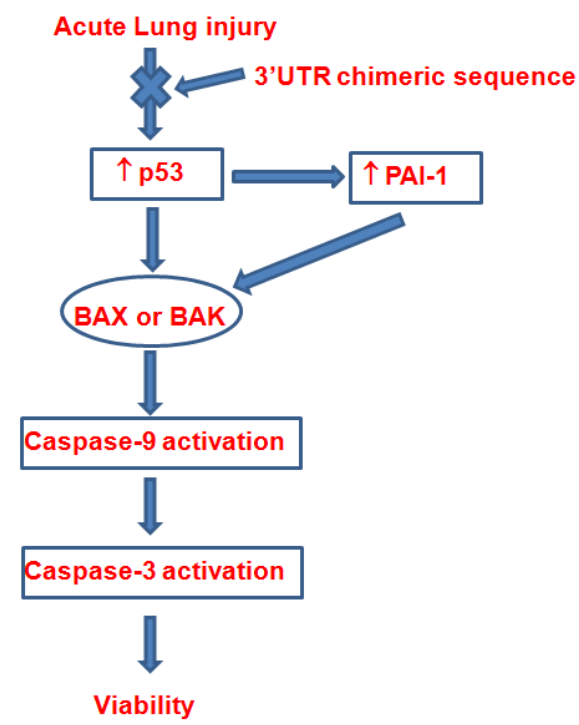

Fig. 3. Mode of action of CSP and 3'UTR chimeric sequence in uPA-deficient mice. (A) In uPA-deficient mice, in response to DNA damage, p53 activates cell death via BAX or BAK activation leading to activation of caspase 9 and initiates the caspase 3 activation. CSP does not have any effect in regulating p53 mediated PAI-1 expression and ATII cell apoptosis since CSP requires uPA to suppress p53 mediated PAI-1 expression and ATII apoptosis. (B) 3'UTR chimeric sequence mitigates p53-mediated induction of PAI-1 expression and ATII cell apoptosis by interfering with p53 and PAI-1 mRNA interaction.

ulate uPA at the mRNA level (Shetty \& Idell 2000). uPA with the help of its receptor uPAR induced the proliferation of alveolar epithelial cells and plays an important role in preventing the development of alveolar fibrosis by protecting alveolar epithelial cells against apoptosis, in addition to its effective function in maintaining alveolar proteolysis (Shetty et al. 2008a). uPA with the help of integrins also induced the phenotypic changes in the ATII cells that are similar to EMT during hypoxia (Shetty et al. 2008a). Thus the regulation of uPA-uPAR-PAI-1 fibrinolytic system in the lung ep- ithelium is mainly through regulation of uPA signaling and cross-talk between uPA and p53, by which uPA can regulate cell viability (Bhandary et al. 2012, 2013, 2015a,b; Shetty et al. 2012).

uPA-deficient mice exposed to BLM are very sensitive to ATII cell apoptosis, while CSP has no diminishing effect on apoptosis (Bhandary et al. 2012, 2013). This shows that $\mathrm{uPA}$ plays a major role in regulating ATII cell apoptosis, and the inability of CSP to downregulate p53 and PAI-1 expression indicate that inhibition of p53 and downstream PAI-1 expression by uPA 
are crucial for CSP to diminish ATII cell apoptosis and ALI (Fig. 3). However, 3'UTR chimeric sequence inhibits PAI-1 expression and ATII cell apoptosis in both wild-type and uPA-deficient mice without suppressing BLM-induced p53 expression (Fig. 3). While CSP attenuates ATII cell apoptosis through uPA-mediated suppression of BLM-induced p53, leading to inhibition of downstream PAI-1 expression, 3'UTR chimeric sequence mitigated p53-mediated induction of PAI-1 expression and ATII cell apoptosis by interfering with p53 and PAI-1 mRNA interaction without affecting BLMinduced ATII cell p53 expression (Bhandary et al. 2012, 2013).

\section{PAI-1 and ALI}

ALI is characterized by deposition of fibrin due to the defect in fibrinolysis, where there is a difference in the balance in proteases and antiproteases (Sapru et al. 2010; Hua et al. 2011). The defect in the fibrinolytic system, which occurs mainly in human and animals with the variety of lung injuries is mediated mainly through increased expression of PAI-1 (Idell 2003; Balsara et al. 2008) and is also induced in the plasma of smokers (Simpson et al. 1997).

Literature suggests that increased PAI-1 contributes to inhibition of apoptosis of neutrophil as well as suppression of phagocytosis of apoptotic neutrophil resulting in accumulation of neutrophil in the injured lungs leading to inflammation (Park et al. 2008; Bhandary et al. 2015b). Coordinated interaction of p53 with PAI-1 mRNA results in reciprocal suppression of uPA and uPAR with an increment in PAI-1 expression, resulting in suppression of survival signals and contributing to epithelial cell apoptosis (Bhandary et al. 2012, 2013, 2015a,b; Shetty et al. 2012) (Fig. 4). Increased AKT phosphorylation and inactivation of phosphatase and tensin homolog support the survival effect of ATII cells and resistance against ATII cell apoptosis (Shetty et al. 2012). Elevated Bcl-X $\mathrm{L}_{\mathrm{L}}$ expression in PAI1 deficient mice also plays important role in complete resistance against ATII cell apoptosis.

\section{Conclusions}

Epithelial cell survival is determined by the regulation of apoptosis in many of the pulmonary diseases. As a consequence of ALI, lung ATII cells undergo apoptosis during normal lung development and maturation. It is important to understand the mechanism, by which these cells resist apoptosis when exposed to certain insult and how these are linked to the airway homeostasis. The mechanisms discussed here will have the significant role in the pathogenic process of all the disorders of epithelial origin. Alveolar epithelial cells with increased p53 and PAI-1 expression are more susceptible to apoptosis, while the mice lacking p53 or PAI-1 (p53-deficient and PAI-1-deficient) resist ATII cell apoptosis, signifying the up-regulation of PAI by p53 is central to the pathogenesis of epithelial injury. Inhibition of apopto-

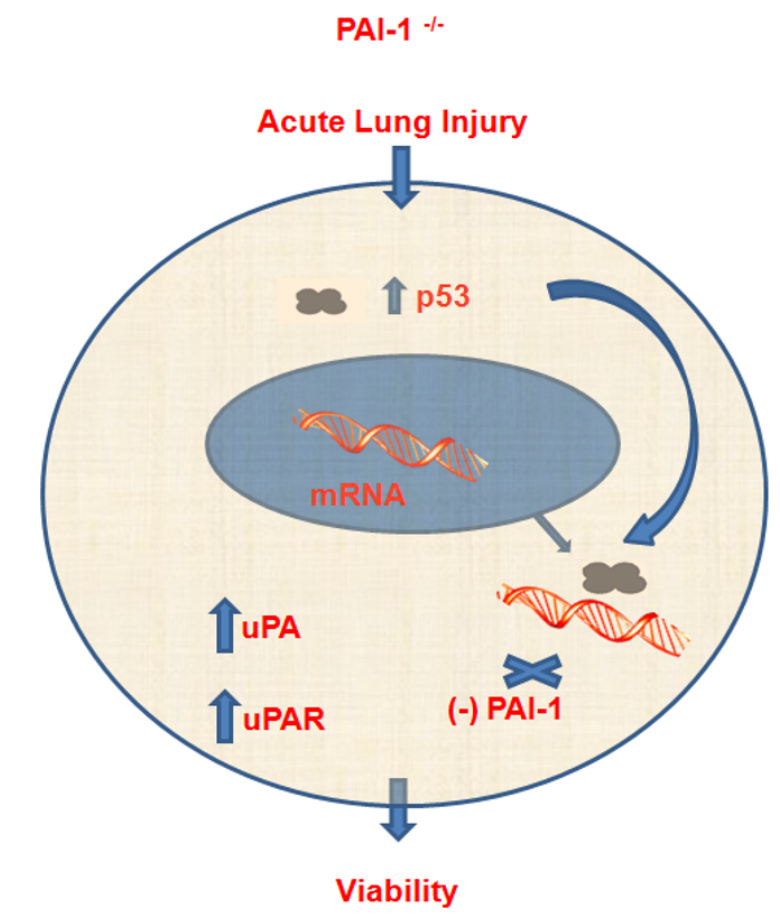

Fig. 4. PAI-1-deficient mice resist ATII cell apoptosis. Regulation of alveolar epithelial cell apoptosis is mediated through cross talk between p53 and components of fibrinolytic system. In PAI-1deficient mice there will be resistance in ATII cell apoptosis since there is no interaction of p53 binding to PAI-1. This results in stabilization of UPA and UPAR and viability of cells.

sis by peptide-based interventions or through 3'UTR chimeric gene sequence may shed some light on the precise understanding of alveolar epithelial cell apoptosis during epithelial injury. An aggressive trial needs to be strategized for the prevention of ALI leading to certain lung diseases.

\section{Acknowledgements}

I sincerely acknowledge Dr. Sreerama Shetty, Professor, Cellular and Molecular Biology, UT Health Northeast for mentoring me in the field of acute lung injury and pulmonary fibrosis. Help from my colleague staff members from UT Health Northeast and Yenepoya Research Centre is highly appreciated.

\section{References}

Alfano D., Franco P., Vocca I., Gambi N., Pisa V., Mancini A., Caputi M., Carriero M.V., Iaccarino I. \& Stoppelli M.P. 2005. The urokinase plasminogen activator and its receptor: role in cell growth and apoptosis. Thromb. Haemost. 93: 205-211.

Balsara R.D. \& Ploplis V.A. 2008. Plasminogen activator inhibitor-1: the double-edged sword in apoptosis. Thromb. Haemost. 100: 1029-1036.

Barazzone C., Belin D., Piguet PF., Vassalli J.D. \& Sappino A.P. 1996. Plasminogen activator inhibitor1 in acute hyperoxic mouse lung injury. J. Clin. Invest. 98: 2666-2673.

Bertozzi P., Astedt B., Zenzius L., Lynch K., LeMaire F., Zapol W. \& Chapman H.A Jr. 1990. Depressed bronchoalveolar urokinase activity in patients with adult respiratory distress syndrome. N. Engl. J. Med. 322: 890-897. 
Bhandary Y.P., Shetty S.K., Marudamuthu A.S., Gyetko M.R., Idell S., Kermani M.G., Shetty R.S., Starcher B.C. \& Shetty S. 2012. Regulation of alveolar epithelial cell apoptosis and pulmonary fibrosis by coordinate expression of components of the fibrinolytic system. Am. J. Physiol. Lung Cell. Mol. Physiol. 302: L463-L473.

Bhandary Y.P., Shetty S.K., Marudamuthu A.S., Ji H.L., Neuenschwander P.F., Boggaram V., Morris G.F., Fu J., Idell S. \& Shetty S. 2013. Regulation of alveolar epithelial injury and lung fibrosis by p53-mediated changes in urokinase and plasminogen activator inhibitor-1. Am. J. Path. 183: 131-143.

Bhandary Y.P., Shetty S.K., Marudamuthu A.S., Fu J., Pinson B.M., Levin J. \& Shetty S. 2015a. Role of p53-fibrinolytic system cross-talk in the regulation of quartz-induced lung injury. Toxicol. Appl. Pharmacol. 283: 92-98.

Bhandary Y.P., Shetty S.K., Marudamuthu A.S., Midde K.K., Ji H.L., Shams H., Idell S., Fu J. \& Shetty S. 2015b. Plasminogen activator inhibitor-1 in cigarette smoke exposure and influenza A virus infection-induced lung injury. Plos One 10: e0123187.

Drapkin P.T., O'Riordan C.R., Yi S.M., Chiorini J.A., Cardella J., Zabner J. \& Welsh M.J. 2000. Targeting the urokinase plasminogen activator receptor enhances gene transfer to human airway epithelia. J. Clin. Invest. 105: 589-596.

Eitzman D.T., McCoy R.D., Zheng X., Fay W.P., Shen T., Ginsburg D. \& Simon R.H. 1996. Bleomycin induced pulmonary fibrosis in transgenic mice that either lack or over express the murine plasminogen activator inhibitor1 gene. J. Clin. Invest. 97: 232-237.

Haraquchi M. 2009. The role of the transcriptional regulator snail in cell detachment, reattachment and migration. Cell. Adh. Migr. 3: 259-263.

Horowitz J.C., Rogers D.S., Simon R.H., Sisson T.H. \& Thannickal V.J. 2008. Plasminogen activation induced pericellular fibronectin proteolysis promotes fibroblast apoptosis. Am. J. Respir. Cell. Mol. Biol. 38: 78-87.

Hua F., Ren W. \& Zhu L. 2011. Plasminogen activator inhibitor type-1 deficiency exaggerates LPS-induced acute lung injury through enhancing Toll-like receptor 4 signaling pathway. Blood Coagul. Fibrinolysis 22: 480-486.

Idell S. 1994. Extravascular coagulation and fibrin deposition in acute lung injury. New Horiz. 2: 566-574.

Idell S. 1995. Coagulation, fibrinolysis and fibrin deposition in lung injury and repair, pp. 743-776. In: Phan S.H. \& Thrall R.S. (eds) Pulmonary Fibrosis. New York, Dekker.

Idell S. 2002. Endothelium and disordered fibrin turnover in the injured lung: newly recognized pathways. Crit. Care Med. 30: S274-S280.

Idell S. 2003. Coagulation, fibrinolysis, and fibrin deposition in acute lung injury. Crit. Care Med. 31: S213-S220.

Jeffery P.K. 2001. Remodeling in asthma and chronic obstructive lung disease. Am. J. Respir. Crit. Care Med. 164: S28-S38.

Lisanti M.P., Tang Z., Scherer P.E., Kubler E., Koleske A.J. \& Sargiacomo M. 1995. Caveolae, transmembrane signalling and cellular transformation. Mol. Membr. Biol. 12: 121-124.

Liu J., Lee P., Galbiati F., Kitsis R.N. \& Lisanti M.P. 2001. Caveolin-1 expression sensitizes fibroblastic and epithelial cells to apoptotic stimulation. Am. J. Physiol. Cell. Physiol. 280: C823-C835.

Miyashita T., Krajewski S., Krajewska M., Wang H.G., Lin H.K., Liebermann D.A., Hoffman B. \& Reed J.C. 1994. Tumor suppressor P53 is a regulator of bcl-2 and bax gene expression in vitro and in vivo. Oncogene9: 1799-1805.

Miyashita T. \& Reed J.C. 1995. Tumor suppressor p53 is a direct transcriptional activator of the human bax gene. Cell 80: 293-299.

Olman M.A., Mackman N., Gladson C.L., Moser K.M. \& Loskutoff D.J. 1995. Changes in procoagulant and fibrinolytic gene expression during bleomycin induced lung injury in the mouse. J. Clin. Invest. 96: 1621-1630.
Park Y.J., Liu G., Lorne E.F., Zhao X. \& Wang J. 2008. PAI-1 inhibits neutrophil efferocytosis. Proc. Natl. Acad. Sci. USA 105: 11784-11789.

Sapru A., Curley M.A., Brady S., Matthay M.A. \& Flori H. 2010. Elevated PAI-1 is associated with poor clinical outcomes in pediatric patients with acute lung injury. Intensive Care Med. 36: $157-163$.

Serrano-Mollar A., Nacher M., Gay-Jordi G., Closa D., Xaubet A. \& Bulbena O. 2007. Intratracheal transplantation of alveolar type II cells reverses bleomycin-induced lung fibrosis. Am. J. Respir. Crit. Care Med. 176: 1261-1268.

Shetty P., Velusamy T., Bhandary Y P., Liu M., Shetty R.S. \& Shetty S. 2008. Urokinase expression by tumor suppressor protein p53: a novel role in mRNA turnover. Am. J. Resp. Cell. Mol. Biol. 39: 364-372.

Shetty S.K., Bhandary Y.P., Marudamuthu A.S., Abernathy D., Veluswamy T., Starcher B. \& Shetty S. 2012. Regulation of airway and alveolar epithelial cell apoptosis by p53 induced PAI-1 during cigarette smoke exposure injury. Am. J. Respir. Cell. Mol. Biol. 47: 474-483.

Shetty S., Gyetko M.R. \& Mazar A.P. 2005. Induction of p53 by urokinase in lung epithelial cells. J. Biol. Chem. 280: 2813328141.

Shetty S. \& Idell S. 2000. Post-transcriptional regulation of urokinase mRNA. Identification of a novel urokinase mRNAbinding protein in human lung epithelial cells in vitro. J. Biol. Chem. 275: 13771-13779.

Shetty S., John J. \& Idell S. 2007a. Fibrosis including fibrinolytic pathways, pp. 101-103. In: Light R.W. \& Lee Y.C.G. (eds) Textbook of Pleural Diseases, $2^{\text {nd }}$ Edition. London: Hodder Arnold.

Shetty S., Padijnayayveetil J., Tucker T., Stankowska D. \& Idell S. 2008a. The fibrinolytic system and the regulation of lung epithelial cell proteolysis, signaling, and cellular viability. Am. J. Physiol. Lung Cell. Mol. Physiol. 295: L967-L975.

Shetty S., Rao G.N., Cines D.B. \& Bdeir K. 2006. Urokinase induces activation of STAT3 in lung epithelial cells. Am. J. Physiol. Lung Cell. Mol. Physiol. 291: L772-L780.

Shetty S., Shetty P., Velusamy T., Idell S., Bhandary Y.P. \& Shetty R.S. 2008b. Regulation of plasminogen activator inhibitor-1 expression by tumor suppressor protein p53. J. Biol. Chem. 283: 19570-19580.

Shetty S., Velusamy T., Shetty P., Idell S., Mazar A.P., Bhandary Y.P. \& Shetty R.S. 2007b. Regulation of urokinase receptor expression by p53: a novel role in stabilization of uPAR mRNA. Mol. Cell. Biol. 27: 5607-5618.

Simpson A.J., Gray R.S., Moore N.R. \& Booth NA. 1997. The effects of chronic smoking on the fibrinolytic potential of plasma and platelets. Br. J. Haematol. 97: 208-213.

Sisson T.H., Hattori N., Xu Y. \& Simon R.H. 1999. Treatment of bleomycin induced pulmonary fibrosis by transfer of urokinase type plasminogen activator genes. Hum. Gene Ther. 10: 2315-2323.

Tiddens H., Silverman M. \& Bush A. 2000. The role of inflammation in airway disease: remodeling. Am. J. Respir. Crit. Care Med. 162: S7-S10.

Wanaski S.P., Ng B.K. \& Glaser M. 2003. Caveolin scaffolding region and the membrane binding region of SRC form lateral membrane domains. Biochemistry 42: 42-56.

Wei Y., Yang X., Liu Q., Wilkins J.A. \& Chapman H.A. 1999. A role of caveolin and the urokinase receptor in integrinmediated adhesion and signaling. J. Cell. Biol. 144: 12851294.

Received June 23, 2016 Accepted October 17, 2016 